Ryo Suminaga - Yasuhiro Takeshima - Kayo Adachi Mariko Yagi · Hajime Nakamura • Masafumi Matsuo

\title{
A novel cryptic exon in intron 3 of the dystrophin gene was incorporated into dystrophin mRNA with a single nucleotide deletion in exon 5
}

Received: December 11, 2001 / Accepted: February 1, 2002

\begin{abstract}
The dystrophin gene, which is mutated in Duchenne muscular dystrophy, is the largest human gene. A full spectrum of the gene transcripts has not been fully elucidated yet, although two cryptic exons have so far been identified in the $5^{\prime}$ region of the dystrophin gene. Here, a novel dystrophin mRNA containing a 62-nucleotide insertion between exons 3 and 4 was identified in lymphocytes from a Japanese Duchenne muscular dystrophy patient with a single nucleotide deletion in exon 5 . The inserted 62nucleotide sequence was found to be homologous to part of intron 3 and it was revealed that the insertion possessed branch point and both acceptor and donor splice site consensus sequences perfectly. Therefore, the 62-bp insertion sequence was considered to be a novel exon and was designated as exon $3 \mathrm{a}$. However, this insertion was not present in the patient's muscle and 12 different normal tissues that were screened. The physiological role of the novel cryptic exon remains to be clarified.
\end{abstract}

Key words Dystrophin · Splicing · Intron · Cryptic exon · Mutation

\section{Introduction}

The human dystrophin gene, which is defective in patients with Duchenne or Becker muscular dystrophy (DMD/ BMD), encodes a protein called dystrophin that is immunohistochemically stained along the plasma membrane of muscle cells. It spans approximately $3000 \mathrm{~kb}$ of the $\mathrm{X}$ chromosome and encodes a 14-kb transcript consisting of

R. Suminaga $\cdot$ K. Adachi $\cdot$ M. Yagi $\cdot$ M. Matsuo $(\bowtie)$ Division of Molecular Medicine, Kobe University Graduate School of Medicine, 7-5-1 Kusunokicho, Chuo-ku, Kobe 650-0017, Japan Tel. +81-78-382-5700; Fax +81-78-382-5719

e-mail: matsuo@kobe-u.ac.jp

Y. Takeshima $\cdot$ H. Nakamura

Department of Pediatrics, Kobe University Graduate School of Medicine, Kobe, Japan
79 exons (Ahn and Kunkel 1993; Nishio et al. 1994). Consequently, more than $99 \%$ of the gene sequence is composed of introns. The extraordinary nature of the dystrophin gene with respect to its intron size and its large number of exons points to several potential characteristics in the processing of dystrophin mRNAs (Surono et al. 1997, 1999; Dwi Pramono et al. 2000). Several dystrophin isoforms are produced in a tissue- or development- specific manner by alternative splicing (Feener et al. 1989; Chelly et al. 1990; Reiss and Rininsland 1994; Barbieri et al. 1996; Torelli and Muntoni 1996). We have identified six novel alternative splicing patterns in the $5^{\prime}$ region of the dystrophin gene in addition to the six already-known patterns, indicating that exon usage in the $5^{\prime}$ region of the dystrophin gene is highly complicated (Surono et al. 1997).

Approximately two thirds of DMD/BMD cases carry deletions or duplications (usually of several kilobases of genomic DNA) in the dystrophin gene. The remaining cases are probably due to small mutations, but the huge size of the dystrophin gene hampers identification of the responsible mutation. Instead of analyzing genomic DNA, dystrophin mRNA has been used as a material for mutation analysis (Roberts et al. 1991), and ectopic dystrophin mRNA expressed in lymphocytes is commonly used because of its easy accessibility. As a result, point mutations scattered on the gene have been disclosed (web site, http:// www.dmd.nl/dmdhome.htm).

Recently, two nonauthentic exons, exon $1 \mathrm{a}$ and exon $2 \mathrm{a}$ from introns 1 and 2, respectively, have been reported to be incorporated into the dystrophin mRNA (Roberts et al. 1993; Dwi Pramono et al. 2000). Because these two transcripts contain an inframe stop codon, no physiological role has been clarified. The insertion of exon 1a between exons 1 and 2 has been observed only in lymphocytes and is identified in half of the dystrophin transcripts (Roberts et al. 1993). This indicates that the environment regulating the splicing of intron 1 in lymphocytes is different from that of muscle cells, where no insertion of exon 1a is observed.

Splicing, wherein introns are removed from pre-mRNA as lariats, proceeds with remarkable accuracy. This process is carried out by spliceosomes, which are multicomponent 
ribonuclear protein complexes. It is well known that consensus sequences at the splice donor and acceptor sites and a branch site of the intron are used for proper splicing (Green 1986). Because the dystrophin gene has an extraordinary structure of huge introns, splicing is conceived to be regulated more sophisticatedly than it is other genes. Recently, exonic splicing enhancer has been shown to be critical for splicing of dystrophin pre-mRNA (Takeshima et al. 1995; Shiga et al. 1997). However, inclusions of intron sequences into dystrophin mRNAs have been reported, the retention of a part of an intron the result of mutations at the splicing consensus sequences (Roest et al. 1996) or the creation of a novel exon from an intron sequence the result of intron mutation (Ikezawa et al. 1998). The latter indicates that creation of splicing consensus sequences located far from the authentic splice site can be recognized as exons by spliceosomes and incorporated into mRNA.

The presence of a splicing abnormality together with a point mutation has been explained as being due to the disruption of splicing consensus sequence or exonic splicing enhancer sequence. Here, we report incorporation of a novel exon (exon 3a) into dystrophin mRNA expressed in lymphocytes from a Japanese DMD case without any mutation at splicing consensus sequences or splicing enhancer sequence, but with a single nucleotide deletion in exon 5 .

\section{Subjects and methods}

\section{Case}

More than $100 \mathrm{DMD} / \mathrm{BMD}$ cases referred to the Kobe University Hospital, Kobe, Japan were enrolled to identify mutations in the dystrophin gene. In nearly half of the cases, large deletions or duplications were identified. The remaining cases were subjected to reverse transcription-polymerase chain reaction (PCR) analysis of dystrophin mRNA prepared from peripheral lymphocytes to identify small mutations. In this study, an 18-year-old Japanese DMD case was examined for mutations of the dystrophin gene. His elder brother was also diagnosed as DMD and died at the age of 17 years as a result of heart failure. In contrast, the current DMD patient's cardiac examination disclosed no abnormality. He showed muscle weakness, and a remarkable elevation of serum creatine kinase level was pointed out at 5 years of age. His muscle weakness progressed and he was wheelchair bound at the age of 11 years. A muscle biopsy done on the biceps muscle showed the absence of dystrophin staining, thus confirming the diagnosis of DMD.

\section{Molecular analysis}

Total RNA was isolated from peripheral lymphocytes or skeletal muscle biopsy samples and cDNA was prepared from total RNA as previously described (Matsuo et al. 1991). To analyze dystrophin cDNA, we amplified a full- length dystrophin transcript as ten separate fragments that overlapped at either end (Roberts et al. 1991). One of these fragments encompassed exons 1 to 11 of the dystrophin mRNA, and a small region encompassing exons 1 to 5 or 1 to 8 was amplified for further study as described (Surono et al. 1997).

To examine the efficiency of activation of exon $3 \mathrm{a}$ in different tissues, we amplified a fragment spanning exon $3 \mathrm{a}$ to exon 5 from cDNA prepared from the total RNA of 12 adult human tissues (skeletal muscle, cardiac muscle, brain, stomach, small intestine, colon, lung, spleen, liver, kidney, testis, and placenta) (Sawady, Tokyo, Japan). cDNA corresponding to $0.125 \mu \mathrm{g}$ of each of the RNA samples was subjected to PCR amplification using the primer set of 5'-TATCAGAATGTAAACCAACATCAT-3' $(\mathrm{C} 3 \mathrm{aF})$ and 5'-TGCCAGTGGAGGATTATATTCCAA3' (C5R).

Human genomic DNA was isolated from lymphocytes of the patient and from a normal individual via previously described methods (Matsuo et al. 1991). PCR amplification of the genomic region encompassing exon $3 \mathrm{a}$ or exon 5 was performed essentially as described previously (Matsuo et al. 1991) using primer sets of 5'-GCCTCCTGGGTTCAAG CAAT-3' (INT3-F), 5'-GCTAAAGCCATCTTACTCTC CAAGG-3'(INT3-R), 5'-CAACTAGGCATTTGGTC TC-3' (g5F), and 5'-TTGTTTCACACGTCAAGGG-3' (g5R), respectively. Genomic regions encompassing exons 3 and 4 were amplified as described earlier (Dwi Pramono et al. 2000).

For DNA sequencing, PCR-amplified products were separated by electrophoresis with low-melting agarose gels. Bands of the amplified products were excised from the gel and the DNA obtained was purified. The purified DNA was subcloned into pT7 vector (Novagen, Madison, WI, USA) and the DNA insert was sequenced using an automatic DNA sequencer (model 373A, Perkin Elmer Applied Biosystems, Norwalk, CT, USA).

\section{Results}

In lymphocytes of the index case, two products were observed, one corresponding to the larger product from the control and the other product having a larger molecular size than the larger control (Fig. 1). Sequencing of the two products from the index case revealed that the smaller one retained exon 1a (162 bp) between exons 1 and 2, as observed in normal lymphocytes (Roberts et al. 1993), but had a single nucleotide A deletion at the 16th nucleotide of exon 5 , corresponding to the 488th nucleotide of the dystrophin cDNA (A488del) (Fig. 2a). This deletion caused a shift in the translational reading frame, resulting in the creation of a stop codon in the 12th codon of exon 5. This nucleotide change was confirmed to be present in the genomic DNA of the index case (data not shown). Furthermore, his mother had the same mutation in the heterozygous state and was identified as a carrier. Because no other mutation was identified in any other coding sequence (data not shown), it 


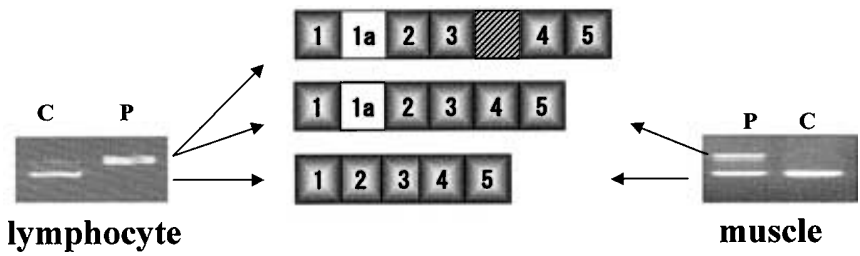

Fig. 1. Amplified products of dystrophin cDNA. The $5^{\prime}$ region of dystrophin mRNA spreading from exons 1 to 5 was amplified from cDNA prepared from lymphocytes (left panel) and from a skeletal muscle biopsy sample (right panel). In lymphocytes, an expected normal-sized product and a larger-sized product were obtained from the normal control (lane $C$ ), while two large-sized products were obtained from the index case but no normal-size product was obtained (lane $P$ ). In skeletal muscle, three bands, two clear and one weak, were visualized, with the weak band found to be nonspecific (lane P). Only one band was visualized from the normal control (lane $C$ ). The exon component of the amplified product is schematically described in the middle

was concluded that this nucleotide deletion was the cause of DMD.

Sequencing of the larger product from the index case disclosed that it contained an unidentified sequence inserted between exons 3 and 4 in addition to the insertion of exon 1a and a mutation of A488 del (Fig. 2b). The inserted sequence was 62 nucleotides (nt) long (Fig. 2b). BLAST search revealed that the inserted sequence was completely homologous to a part of intron 3 and that its $5^{\prime}$ and $3^{\prime}$ ends were present $3045 \mathrm{nt}$ downstream of the $3^{\prime}$ end of exon 3 and $1761 \mathrm{nt}$ upstream of exon 4, respectively (GenBank AL096699) (Fig. 2c). Further examination of the nucleotide sequence around the identical region of intron 3 revealed that the AG and GT dinucleotides that form consensus sequences at the splice acceptor and donor sites of all introns, respectively, were present immediately adjacent to the $5^{\prime}$ and $3^{\prime}$ ends of the 62-bp sequence (Fig. 2c). Shapiro's scores for the splice acceptor and donor sites were 70.3 and 79.9, respectively (Shapiro and Senapathy 1987). Furthermore, the sequence TCCTGAT, with a match to the branch point consensus sequence (YNYTRAC, $\mathrm{Y}=\mathrm{C}$ or $\mathrm{T} ; \mathrm{R}=\mathrm{G}$ or $\mathrm{A} ; \mathrm{N}=$ any base), was identified $110 \mathrm{nt}$ upstream of the putative splice acceptor site. A polypyrimidine tract was also identified between the putative branch point and the splice acceptor site. Therefore, the 62-bp insertion sequence was considered to maintain all characteristics typical of a genomic exon and was named exon 3a (Fig. 2c).

Because the exon 3a insertion was postulated to be due to a genomic mutation, the intron region covering the inserted $62 \mathrm{nt}$ and its flanking parts was sequenced (Fig. 2c). However, it was found that $62 \mathrm{nt}$ of the insertion and the nucleotide sequences $210 \mathrm{nt}$ upstream and $100 \mathrm{nt}$ downstream were completely identical to the reported sequence (GenBank AL096699) (data not shown). Genomic sequences encompassing exons 3 or 4 were also analyzed but no nucleotide change responsible for the splicing error was identified. It was thus concluded that the incorporation of exon 3a was not due to a genomic mutation.

To investigate whether the same 62-nt sequence was also incorporated into the dystrophin transcript expressed in the a.

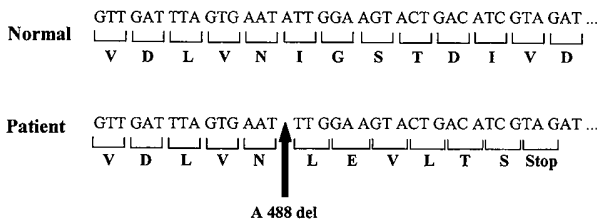

b.
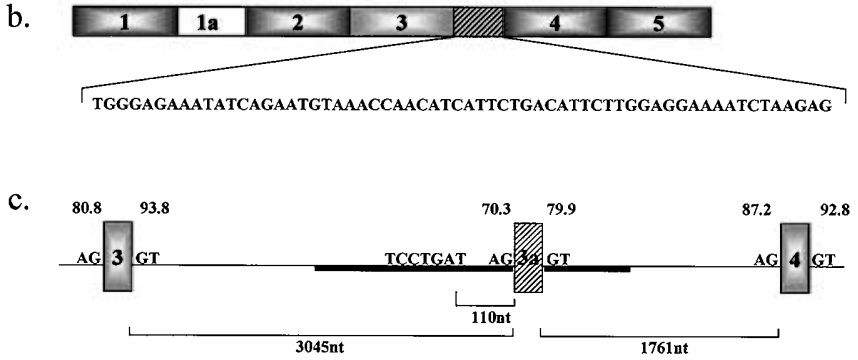

Fig. 2a-c. Sequence of the dystrophin cDNA of the index case. a Sequence of exon 5. The A nucleotide at $488 \mathrm{nt}$ was missing (A488del) and it resulted in a TAG stop codon at the seventh downstream codon (below). b Schematic description of the largest product. The insertion of exon 1a and a nucleotide deletion (A488del) were present. Additionally, the 62-bp long sequence was inserted between exons 3 and 4 and its sequence is described below. $\mathbf{c}$ Schematic description of location of exon 3a. The inserted 62-nt sequence, exon 3a, was present in intron 3 , maintaining both the consensus splice sites and a branch site. The distance from exon 3a is shown in brackets. The numbers over the exons indicate the Shapiro's score for the respective sites. Boxes and bars indicate exons and introns, respectively. Bars indicate introns that maintain GT and AG dinucleotides at the $5^{\prime}$ and $3^{\prime}$ end of its sequence. Bold bars indicate the sequenced intron regions in the index case. The figure is not drawn to scale

patient's skeletal muscle, we amplified the fragment spanning exons 1 to 5 from cDNA prepared from the patient's muscle (Fig. 1). Three bands were visualized, with each band subjected to sequencing. The smallest product consisted of exons 1 to 5 and was considered the normal splicing product. The largest product contained exon 1a between exons 1 and 2 . The weak band between the two clear bands (Fig. 1) was found to be a nonspecific product. Because exon 1a is not incorporated into the dystrophin mRNA expressed in normal skeletal muscle, it was suggested that regulated splicing of the dystrophin pre-mRNA was disrupted in the patient's muscle. To examine a trace amount of transcript containing exon $3 \mathrm{a}$, we subsequently subjected the amplified product to PCR amplification using a set of primers located in the inserted sequence and exon 5 . However, no product was obtained (data not shown).

The incorporation of intron 3 was examined in 12 different normal tissues by amplifying a fragment extending from the inserted sequence to exon 5. However, the product containing exon $3 \mathrm{a}$ was not obtained from any of the 12 tissues (data not shown). These results indicate that exon 3a was not used in any normal adult tissue. Although we have analyzed dystrophin mRNAs expressed in lymphocytes from more than $100 \mathrm{DMD} / \mathrm{BMD}$ cases with deletion mutations or point mutations in the dystrophin gene (Matsuo et al. 1991; Hagiwara et al. 1994; Shiga et al. 1997; Wibawa et al. 2000), a similar-sized fragment as identified in the present case has never been observed (data not shown). Dystrophin mRNA containing exon 3a could not be identi- 
Patient

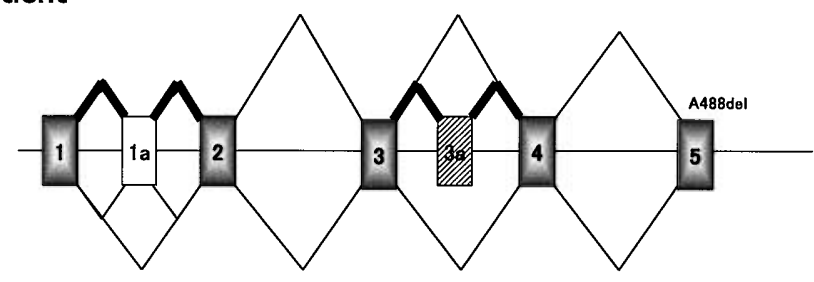

Normal

Fig. 3. Splicing patterns of the dystrophin transcript in lymphocytes. Splicing reactions in lymphocytes of the patient and control are indicated as diagonal lines above and below the schematic representation of the gene, respectively. The bold diagonal lines indicate the activated splicing process. The boxes and the numbers in the boxes indicate exons and their corresponding numbers

fied in the lymphocytes of his mother, a carrier of A488 del (data not shown). This suggests that the use of exon $3 \mathrm{a}$ was very specific to the index case.

Protein-coding ability was subsequently analyzed on the novel exon. Because the transcript identified in this study contained not only the novel exon $3 \mathrm{a}$, but also exon $1 \mathrm{a}$, a premature stop codon appeared on the exon 1a-encoded region. We decided, therefore, that the novel mRNA nonfunctional for producing protein.

In conclusion, novel exon $3 \mathrm{a}$ was identified as being inserted into dystrophin mRNA expressed in lymphocytes from a DMD case who had a genomic mutation of A488del in exon 5 of the dystrophin gene. The physiological role of exon 3 a remains to be clarified.

\section{Discussion}

In this report, a novel mutation of the dystrophin gene (A488del) was identified in a Japanese case of DMD. Remarkably, splicing abnormalities of two upstream introns were also disclosed in his lymphocytes: incorporation of exons 1a and 3a into dystrophin mRNA (Fig. 3). Exon 1a, which is incorporated in half of the dystrophin mRNA from normal lymphocytes (Roberts et al. 1993), was incorporated into all mRNA in lymphocytes of the index case. Furthermore, exon 3a was found to be inserted in half of the dystrophin mRNA (Fig. 1). Exon 1a, which never incorporated in his muscle, was observed to be inserted in a small fraction of the dystrophin mRNA. In contrast to lymphocytes, no mRNA containing exon 3a was identified in his muscle.

Exon 3a was first found to be inserted into mRNA, but this incorporation was both tissue- and mutation-specific; the insertion event was limited to mRNA expressed in lymphocytes of one particular DMD patient who had a single nucleotide deletion in exon 5 (A488del) (Fig. 2a). Exon 3a was found to maintain the consensus sequences for splicing but was not recognized as an exon in usual splicing environments (Fig. 3). Exon 3a is considered a weak exon based on the following points: (1) the branch point is present $110 \mathrm{nt}$ upstream of the splice acceptor site of exon 3a. Usually it is present $20-30 \mathrm{nt}$ upstream of the splice acceptor site. The very long tract between a branch point and the $3^{\prime}$ splice acceptor site may hamper the function of the U2AF35 nuclear protein, a branch point-binding protein, in recognizing the branch site. (2) A purine-rich sequence within the exon sequence is not present in the inserted $62 \mathrm{nt}$. In separate studies, we have shown that some dystrophin exons have purine-rich sequences that function as exonic splicing enhancer sequences (Takeshima et al. 1995; Shiga et al. 1997). Therefore, it was supposed that incorporation of exon 3a was activated by changes in the splicing environment.

Splicing errors of dystrophin transcripts have been reported in mutations of the splicing consensus sequences or exonic splicing enhancer sequences (Matsuo et al. 1991; Hagiwara et al. 1994; Shiga et al. 1997; Wibawa et al. 2000). The incorporation of the middle part of intron sequences has been reported in the creation of a consensus sequence by a single-nucleotide change in the dystrophin gene (Ikezawa et al. 1998). In the index case, we could not identify any mutation responsible for splicing errors. One particular nucleotide change in the index case was an A488 del in exon 5. This nucleotide change was supposed to trigger a splicing error, but we are not able to draw any conclusion about whether splicing abnormalities identified in this report were due to mutations in exon 5. More cases with nonsense mutations in exon 5 of the dystrophin gene (Nigro et al. 1994) are needed to examine this supposition.

The most remarkable finding in the index case was an activated incorporation of exon 1a; all dystrophin mRNA in lymphocytes contained exon 1a and a small proportion of the dystrophin mRNA in his skeletal muscle also contained exon 1a (Fig. 1). These observations suggest that environmental factors that are necessary for proper splicing of intron 1 or exon 1a were disrupted in the index case. There are two possible explanations for double-splicing abnormalities of introns located upstream of the mutation site: (1) RNA secondary structural change and (2) spliceosomal program change. It has been reported that regulation of splicing is due, at least in part, to a secondary structure of the primary transcript (Libri et al. 1991). In genomic DNA, exon 5 is separated roughly $50 \mathrm{~kb}$ and $200 \mathrm{~kb}$ from introns 1 and 3, respectively. Although the secondary structure of pre-mRNA or mRNA may function to bring these separate portions near, it is not plausible that a mutation far downstream can affect the splicing of remote upstream introns, considering that splicing proceeds cotranscriptionally (Bauren and Wieslander 1994). Therefore, the first possibility does not seem likely.

In the spliceosome, more than 30 distinct proteins have been shown to be present (Kramer 1996). It has been reported that different regulatory programs for splicing run concurrently within the same cell, suggesting that the production of different alternatively spliced pre-mRNAs is regulated by distinct programs that use different sets of cis elements and trans acting factors (Cooper and Mattox 1997). Certain factors play active roles in both constitutive splicing and regulation of alternative splicing (Lopez 1998). 
The disruption of regulated mRNA processing has emerged as a cellular function that is disturbed during neoplastic transformation of cells (Sager 1997; Jang et al. 2000). Singlenucleotide mutations have been reported to change splicing, even though it is located outside the splicing consensus sequence (Gibson et al. 1993; Dietz 1997; Liu et al. 1997; Hoffmeyer et al. 1998). In nonsense mutations, skipping of the exon containing the nonsense codon was described. An example of a remote effect on downstream splicing has been reported in a splice site mutation of the cholesteryl ester transfer protein gene (Sakai et al. 1996). Remarkably, inhibition of upstream intron splicing was described in a nonsense mutation of the immunoglobulin $\mathrm{k}$ gene (Aoufouchi et al. 1996). In dystrophin, secondary abnormality of splicing has been reported in exon deletion mutations (Matsuo et al. 1991; Roberts et al. 1994). In any event, our results suggest that the environment of splicing has been perturbed in our patient's lymphocytes and this led to the incorporation of exon 3a (Fig. 3).

It has been suggested that large introns may be removed by a process in which smaller sections are first extracted via intermediate splicing events. Recently, the stepwise removal of a large intron was first demonstrated in the Drosophila ultrabothorax transcript by resplicing at the junction between certain joined exons (Hatton et al. 1998). Exon 3a, which is embedded in a 4.7-kb long intron 3, may be a remnant of such stepwise removal intron. Further study is needed to clarify this possibility.

In Duchenne muscular dystrophy, there is a wide variety of clinical phenotypes with involvement of skeletal and heart muscles and mental activities. The possibility that alternative splicing could modify the clinical phenotype of dystrophinopathy by editing the translational reading frame has been pointed out (Chelly et al. 1991; Winnard et al. 1993; Shiga et al. 1997). It has long been proposed that the cryptic start codon in exon 8 of the dystrophin transcript is activated and that its translation product modifies the clinical phenotype (Gangopadhyay et al. 1992). The incorporation of exon 3a may have a role in the stabilization of the mRNA that has a restart codon in exon 8 and may explain the different clinical phenotype of DMD (Nigro et al. 1994).

Acknowledgments We thank Dr. Lai Poh San for advice and for critically reading the manuscript. We acknowledge Ms. N. Kageyama for her secretarial help. This work was supported by grants from the Ministry of Education, Science and Culture of Japan and the Research Grant (8A-1) for Nervous and Mental Disorders from the Ministry of Health and Welfare of Japan.

\section{References}

Ahn AH, Kunkel LM (1993) The structural and functional diversity of dystrophin. Nat Genet 3:283-291

Aoufouchi S, Yelamos J, Milstein C (1996) Nonsense mutations inhibit RNA splicing in a cell-free system: recognition of mutant codon is independent of protein synthesis. Cell 85:415-422

Barbieri AM, Soriani N, Ferlini A, Michelato A, Ferrari M, Carrera P (1996) Seven novel additional small mutations and a new alternative splicing in the human dystrophin gene detected by heteroduplex analysis and restricted RT-PCR heteroduplex analysis of illegitimate transcripts. Eur J Hum Genet 4:183-187

Bauren G, Wieslander L (1994) Splicing of Balbiani ring 1 gene premRNA occurs simultaneously with transcription. Cell 76:183-192

Chelly J, Hamard G, Koulakoff A, Kaplan J-C, Kahn A, BerwaldNetter Y (1990) Dystrophin gene transcribed from different promoters in neuronal and glial cells. Nature 344:64-65

Chelly J, Gilgenkrantz H, Hugnot J, Hamard G, Lambert M, Recan D, Akli S, Cometto M, Kahn A, Kaplan J (1991) Illegitimate transcription: application to the analysis of truncated transcripts of the dystrophin gene in nonmuscle cultured cells from Duchenne and Becker patients. J Clin Invest 88:1161-1166

Cooper TA, Mattox W (1997) The regulation of splice-site selection, and its role in human disease. Am J Hum Genet 61:259-266

Dietz HC (1997) Nonsense mutations and altered splice-site selection. Am J Hum Genet 60:729-730

Dwi Pramono ZA, Takeshima Y, Surono A, Ishida T, Matsuo M (2000) A novel cryptic exon in intron 2 of the human dystrophin gene evolved from an intron by acquiring consensus sequences for splicing at different stages of anthropoid evolution. Biochem Biophys Res Commun 267:321-328

Feener CA, Koenig M, Kunkel LM (1989) Alternative splicing of human dystrophin mRNA generates isoforms at the carboxy terminus. Nature 338:509-511

Gangopadhyay SB, Sherratt TG, Heckmatt JZ, Dubowitz V, Miller G, Shokeir M, Ray PN, Strong PN, Worton RG (1992) Dystrophin in frameshift deletion patients with Becker muscular dystrophy. Am J Hum Genet 51:562-570

Gibson R, Hajiapour A, Murer-Orland M, Buchwald M, Mathew C (1993) A nonsense mutation and exon skipping in the Fanconi anaemia group C gene. Hum Mol Genet 2:797-799

Green MR (1986) Pre-mRNA splicing. Annu Rev Genet 20:671-708

Hagiwara Y, Nishio H, Kitoh Y, Takeshima Y, Narita N, Wada H, Yokoyama M, Nakamura H, Matsuo M (1994) A novel point mutation $\left(\mathrm{G}^{-1}\right.$ to $\left.\mathrm{T}\right)$ in a $5^{\prime}$ splice donor site of intron 13 of the dystrophin gene results in exon skipping and is responsible for Becker muscular dystrophy. Am J Hum Genet 54:53-61

Hatton AR, Subramaniam V, Lopez AJ (1998) Generation of alternative Ultrabithorax isoforms and stepwise removal of a large intron by resplicing at exon-exon junctions. Mol Cell 2:787-796

Hoffmeyer S, Nurnberg P, Ritter H, Fahsold R, Leistner W, Kaufmann D, Krone W (1998) Nearby stop codons in exons of the neurofibromatosis type 1 gene are disparate splice effectors. Am J Hum Genet 62:269-277

Ikezawa M, Minami N, Takahashi M, Goto Y, Miike T, Nonaka I (1998) Dystrophin gene analysis on 130 patients with Duchenne muscular dystrophy with a special reference to muscle mRNA analysis. Brain Dev 20:165-168

Jang JH, Shin KH, Park YJ, Lee RJ, McKeehan WL, Park JG (2000) Novel transcripts of fibroblast growth factor receptor 3 reveal aberrant splicing and activation of cryptic splice sequences in colorectal cancer. Cancer Res 60:4049-4052

Kramer A (1996) The structure and function of proteins involved in mammalian pre-mRNA splicing. Annu Rev Biochem 65:367-409

Libri D, Piseri A, Fiszman MY (1991) Tissue-specific splicing in vivo of the beta-tropomyosin gene: dependence on an RNA secondary structure. Science 252:1842-1845

Liu W, Qian C, Francke U (1997) Silent mutation induces exon skipping of fibrillin-1 gene in Marfan syndrome. Nat Genet 16:328-329

Lopez AJ (1998) Alternative splicing of pre-mRNA: developmental consequences and mechanisms of regulation. Annu Rev Genet 32:279-305

Matsuo M, Masumura T, Nishio H, Nakajima T, Kitoh Y, Takumi T, Koga J, Nakamura H (1991) Exon skipping during splicing of dystrophin mRNA precursor due to an intraexon deletion in the dystrophin gene of Duchenne muscular dystrophy kobe. J Clin Invest 87:2127-2131

Nigro V, Nigro G, Esposito MG, Comi LI, Molinari AM, Puca GA, Politano L (1994) Novel small mutations along the $D M D / B M D$ gene associated with different phenotypes. Hum Mol Genet 3:1907-1908 Nishio H, Takeshima Y, Narita N, Yanagawa H, Suzuki Y, Ishikawa Y, Minami R, Nakamura H, Matsuo M (1994) Identification of a novel first exon in the human dystrophin gene and of a new promoter located more than $500 \mathrm{~kb}$ upstream of the nearest known promoter. J Clin Invest 94:1037-1042 
Reiss J, Rininsland F (1994) An explanation for the constitutive exon 9 cassette splicing of the DMD gene. Hum Mol Genet 3:295-298

Roberts RG, Barby TF, Manners E, Bobrow M, Bentley DR (1991) Direct detection of dystrophin gene rearrangements by analysis of dystrophin mRNA in peripheral blood lymphocytes. Am J Hum Genet 49:298-310

Roberts RG, Bentley DR, Bobrow M (1993) Infidelity in the structure of ectopic transcripts: a novel exon in lymphocyte dystrophin transcripts. Hum Mutat 2:293-299

Roberts RG, Gardner RJ, Bobrow M (1994) Searching for the 1 in 2,400,000: a review of dystrophin gene point mutations. Hum Mutat $4: 1-11$

Roest PAM, Bout M, van der Tuijn AC, Ginjaar IB, Bakker E, Hogervorst FBL, van Ommen GJB, Den Dunnen JT (1996) Splicing mutations in DMD/BMD detected by RT-PCR/PTT: detection of a 19AA insertion in the cysteine rich domain of dystrophin compatible with BMD. J Med Genet 33:935-939

Sager R (1997) Expression genetics in cancer: shifting the focus from DNA to RNA. Proc Natl Acad Sci USA 94:952-955

Sakai N, Santamarina-Fojo S, Yamashita S, Matsuzawa Y, Brewer HB $\mathrm{Jr}$ (1996) Exon 10 skipping caused by intron 10 splice donor site mutation in cholesteryl ester transfer protein gene results in abnormal downstream splice site selection. J Lipid Res 37:2065-2073

Shapiro MB, Senapathy P (1987) RNA splice junctions of different classes of eukaryotes: sequence statistics and functional implications in gene expression. Nucleic Acids Res 15:7155-7174

Shiga N, Takeshima Y, Sakamoto H, Inoue K, Yokota Y, Yokoyama M, Matsuo M (1997) Disruption of the splicing enhancer sequence within exon 27 of the dystrophin gene by a nonsense mutation induces partial skipping of the exon and is responsible for Becker muscular dystrophy. J Clin Invest 100:2204-2210

Surono A, Takeshima Y, Wibawa T, Pramono ZA, Matsuo M (1997) Six novel transcripts that remove a huge intron ranging from 250 to $800 \mathrm{~kb}$ are produced by alternative splicing of the $5^{\prime}$ region of the dystrophin gene in human skeletal muscle. Biochem Biophys Res Commun 239:895-899

Surono A, Takeshima $\mathrm{Y}$, Wibawa $\mathrm{T}$, Ikezawa $\mathrm{M}$, Nonaka I, Matsuo M (1999) Circular dystrophin RNAs consisting of exons that were skipped by alternative splicing. Hum Mol Genet 8:493500

Takeshima Y, Nishio H, Sakamoto H, Nakamura H, Matsuo M (1995) Modulation of in vitro splicing of the upstream intron by modifying an intra-exon sequence which is deleted from the dystrophin gene in dystrophin Kobe. J Clin Invest 95:515-520

Torelli S, Muntoni F (1996) Alternative splicing of dystrophin exon 4 in normal human muscle. Hum Genet 97:521-523

Wibawa T, Takeshima Y, Mitsuyoshi I, Wada H, Surono A, Nakamura H, Matsuo M (2000) Complete skipping of exon 66 due to novel mutations of the dystrophin gene was identified in two Japanese families of Duchenne muscular dystrophy with severe mental retardation. Brain Dev 22:107-112

Winnard AV, Klein CJ, Coovert DD, Prior T, Papp A, Snyder P, Bulman DE, Ray PN, McAndrew P, King W, Moxley RT, Mendell JR, Burghes AHM (1993) Characterization of translational frame exception patients in Duchenne/Becker muscular dystrophy. Hum Mol Genet 2:737-744 